\title{
BMJ Open Using telemedicine to support care for people with type 2 diabetes mellitus: a qualitative analysis of patients' perspectives
}

Jun Yang Lee, ${ }^{1,2}$ Carina Ka Yee Chan, ${ }^{3}$ Siew Siang Chua, ${ }^{4}$ Thomas Paraidathathu, ${ }^{4}$ Kenneth Kwing-Chin Lee, ${ }^{1}$ Christina San San Tan, ${ }^{5}$ Nazrila Nasir, ${ }^{6}$ Shaun Wen Huey Lee (D) ${ }^{1,4}$

To cite: Lee JY, Chan CKY, Chua SS, et al. Using telemedicine to support care for people with type 2 diabetes mellitus: a qualitative analysis of patients'

perspectives. BMJ Open 2019;9:e026575. doi:10.1136/ bmjopen-2018-026575

- Prepublication history for this paper is available online. To view these files, please visit the journal online (http://dx.doi org/10.1136/bmjopen-2018026575).

Received 10 September 2018 Revised 16 September 2019 Accepted 27 September 2019

\section{Check for updates}

(c) Author(s) (or their employer(s)) 2019. Re-use permitted under CC BY-NC. No commercial re-use. See rights and permissions. Published by BMJ.

${ }^{1}$ School of Pharmacy, Monash University - Malaysia Campus, Bandar Sunway, Malaysia

${ }^{2}$ SEGi University Kota

Damansara, Petaling Jaya,

Malaysia

${ }^{3}$ La Trobe University College of Science Health and Engineering, Bundoora, Victoria, Australia

${ }^{4}$ School of Pharmacy, Taylor's University - Lakeside Campus, Subang Jaya, Malaysia ${ }^{5} \mathrm{HELP}$ International Corporation Bhd, Kuala Lumpur, Malaysia

${ }^{6}$ Kementerian Kesihatan

Malaysia, Putrajaya, Malaysia

Correspondence to

Shaun Wen Huey Lee;

shaun.lee@monash.edu

\section{ABSTRACT}

Objective Telemedicine has been promoted as an economical and effective way to enhance patient care, but its acceptance among patients in low-income and middle-income countries is poorly understood. This study is aimed to explore the experiences and perspectives of people with type 2 diabetes mellitus that used telemedicine to manage their condition.

Design In-depth and focus group interviews were conducted with participants who have engaged in telemedicine. Questions included were participants' perception on the programme being used, satisfaction as well as engagement with the telemedicine programme. All interviews and focus groups were audio-recorded and transcribed verbatim. Data were analysed using a thematic approach

Participants and setting People with type 2 diabetes $(n=48)$ who participated in a randomised controlled study which examined the use of telemedicine for diabetes management were recruited from 11 primary care clinics located within the Klang Valley.

Results Twelve focus groups and two in-depth interviews were conducted. Four themes emerged from the analysis: (1) generational difference; (2) independence and convenience, (3) sharing of health data and privacy and (4) concerns and challenges. The main obstacles found in patients using the telemedicine systems were related to internet connectivity and difficulties experienced with system interface. Cost was also another significant concern raised by participants. Participants in this study were primarily positive about the benefits of telemedicine, including its ability to provide real-time data and disease monitoring and the reduction in clinic visits.

Conclusion Despite the potential benefits of telemedicine in the long-term care of diabetes, there are several perceived barriers that may limit the effectiveness of this technology. As such, collaboration between educators, healthcare providers, telecommunication service providers and patients are required to stimulate the adoption and the use of telemedicine.

\section{TRIAL REGISTRATION NUMBER} NCT0246680.

\section{INTRODUCTION}

Diabetes is a major health concern worldwide with a global prevalence of 415 million
Strengths and limitations of this study

- The study followed the Standards for Reporting Qualitative Research recommendations.

- Focus group discussion allowed for the exchange of opinions relating to the use of telemedicine.

- The views and experiences with telemedicine were documented from the patients' perspectives instead of the healthcare providers' perspective.

- The study was conducted in an urban setting in Malaysia. The implications related to other populations are unknown.

in 2015 rising to 642 million in $2040 .{ }^{1}$ The prevalence of diabetes varies considerably between different global regions, but the epicentre of the disease is currently in the Western Pacific and South-East Asia regions, with 159 million and 82 million individuals with diabetes, respectively. ${ }^{2}$ Self-monitoring of blood glucose (SMBG) is considered to be a key component in the treatment of diabetes, and is widely recommended in clinical practice guidelines, irrespective of subsequent treatment strategy. ${ }^{3-7}$ SMBG is often used as an early indicator for detecting hypoglycaemia, monitoring severe hyperglycaemia and encouraging physical activity as well as improving diet control. The long-term individualised patient data are often used to inform treatment regimens. ${ }^{8}$

Despite these potential benefits, there is controversy regarding the efficacy of SMBG, especially in non-insulin dependent type 2 diabetes, due to its cost, uncertainty with frequency of testing as well as the impact on patient's general health and well-being. ${ }^{9-11}$ Studies have shown that the use of enhanced SMBG (where there are high levels of engagement between patients and clinicians when interpreting a patient's SMBG values) has 
resulted in better maintenance of glycated haemoglobin (HbA1c) levels when compared with regular SMBG. ${ }^{42-14}$

The application of telemedicine in delivering various aspects of health information from the information on the prevention of disease to the monitoring of medical conditions as well as regular medical care for diabetes management has been suggested as an innovative solution to improve diabetic patient care. ${ }^{15-17}$ Telemedicine is being viewed as a useful tool to assist patients in managing diabetes, ${ }^{161819}$ as it facilitates the communication of accurate and reliable data ${ }^{20}$ between patients and their healthcare providers. It also empowers patients ${ }^{15}$ attitude and behaviour ${ }^{191}$ towards a healthier lifestyle while providing them with an outlook for better glycaemic control. ${ }^{22}$ These telemedicine services can be categorised into either synchronous (real-time), asynchronous (whereby data are stored and forwarded subsequently) and continuous (remote monitoring). In diabetes care, various forms of telemedicine services have been examined. These include the use of mobile phones, text messages, email, e-health portals, videoconferences as well as remote monitoring devices.

Implementation of a telemedicine service in conventional care is a complex process. While evidence has shown that telemedicine may expand the boundaries of healthcare to a larger population and may offer person-centred care, ${ }^{23}$ there are still examples where patients resist these changes. ${ }^{24}{ }^{25}$ One of the major limitations of existing literature is the lack of studies which have examined the perspectives of patients in low-income to middle-income countries such as Malaysia, where telemedicine is now being used. Earlier published work on this topic has focused primarily on the views of physicians rather than the patients' perspective. ${ }^{26}{ }^{27}$ There was a limited understanding of the key factors faced by patients using telemedicine as most of these studies have focused primarily on its utilisation rates. ${ }^{25} 28$ Furthermore, very little is known as to how empirically supported interventions can be transferred or implemented in resource-constrained countries, that is, in most developing countries. Understanding the behaviour among various levels of stakeholders is an important component of successful implementation research. ${ }^{29}$

As patients' experience with telemedicine may be a major reason in determining the uptake of this system, we conducted a qualitative study to explore patients' views and experiences of telemedicine for diabetes management in Malaysia. The study focused on the patients' perspective in context which was necessary to ensure the successful delivery and implementation of a telemedicine programme for diabetes.

\section{MATERIALS AND METHODS}

\section{Study design}

The present qualitative study was part of a larger multicentre cluster randomised controlled study (Intervention for Diabetes with Education, Advancement and Support

\section{Box 1 IDEAS study}

- The Intervention for Diabetes with Education, Advancement and Support (IDEAS) study is a cluster randomised controlled study to evaluate the use of telemedicine to improve diabetes care. Participants in the telemedicine group were instructed to measure their blood glucose at least twice weekly (one fasting and one non-fasting) or more frequent as recommended by physician. These blood glucose readings will be transmitted via a Bluetooth technology to the participants' mobile phone to a remote secure server. Participants and their physicians were able to access the records on the server. Advice on lifestyle modification, any potential changes in medication, who and how to contact their healthcare providers were also given monthly during the study. A researcher also checked participants' results weekly and initiate intervention if needed (eg, medication changes, counselling) with the consent of the attending physician.

(IDEAS) ) conducted between April 2015 and June 2017, which examined the impact of a telemedicine programme for people with type 2 diabetes (box 1). ${ }^{30} 31$ This nested study design allowed the investigators to explore the perceived obstacles and incentives patients experienced when using telemedicine in the management of their type 2 diabetes.

\section{Participants and setting}

Participants were recruited from 11 primary care clinics located within the Klang Valley, which is part of the Malaysian Ministry of Health's primary care clinic network. These clinics serve the districts of Klang and Petaling, which provide care for approximately 2.56 million individuals. These individuals are considered to be representative of primary care clinics in Malaysia.

This qualitative study adopted a non-probability sampling method, where sampling was not guided by the idea of random selection or statistical representation. Nevertheless, we aimed to cover as broad a spectrum of participants who had experienced using telemedicine. We invited participants who had completed the IDEAS study to participate in the current qualitative study. Participants included those who: (1) had been diagnosed with type 2 diabetes for at least 6 months; (2) aged between 18 and 75 years; (3) had regular access to the Internet; (4) had HbA1c levels between $7.5 \%$ and $11.0 \%$ and (5) were randomised into the intervention arm of the IDEAS study. ${ }^{30}$ Participants were excluded if they had no experience in using telemedicine devices or had dropped out of the IDEAS study. ${ }^{31}$ All potentially eligible participants were contacted by telephone and invited to participate in the study. Participants were briefed on the purpose, procedure and voluntary nature of the study and were allowed to clarify their concerns. A separate information sheet was given to the participants and written informed consent was obtained prior to the start of the study. To accommodate participants' schedules, all focus group interviews were conducted when participants visited the clinics for their follow-up sessions (at weeks 4, 12, 24 or 
Table 1 The interview guide topic areas and key questions

\begin{tabular}{ll}
\hline Topic area & Key questions used in interviews \\
Diabetes & How would you describe your experience so far with diabetes? \\
& Who and how much support do you have to cope with your conditions now? \\
Telemedicine & What are some of the most important treatment needs for a patient with diabetes? \\
& How do you think the system was able to affect your diabetes management? \\
& How do you think others will accept this system? \\
& What would you think are some areas where the system was good and where are the areas it can be \\
& improved? \\
& What do you think about using this system for the long term? \\
& What would be some of your concerns (if any) if this was to be used? \\
& How was your interaction with your healthcare professional with the device?
\end{tabular}

48) with their physicians. In the event, a participant was not able to attend a focus group session, so an individual in-depth interview was conducted at their home. Study participants were given a RM50 (approximately US\$12) voucher for their participation.

\section{Interview schedule}

A semistructured interview was used in all the focus groups, and this was based on the topic areas and key questions outlined in table 1 . The topic guide aimed to capture the experiences of patients in using telemedicine and was developed based on relevant literature. ${ }^{11} 1324$

The interviews were facilitated by the first author (JYL) and conducted either in Malay or English. Each interview lasted between 35 and $44 \mathrm{~min}$ in a private room in the clinic. All interview sessions were digitally recorded, transcribed verbatim immediately after each interview and checked for accuracy by SWHL. Interviews were conducted to explore for emerging themes with simultaneous analysis of data until data saturation was achieved, when no new themes or codes were identified.

\section{Data analysis}

The NVivo software V.11 (QSR International Pty Ltd) was used to organise and code the data for thematic analysis, using both an inductive and deductive approach. ${ }^{32}$ All transcripts were read several times to familiarise with the data. Transcripts were translated from Malay language to English by an independent research assistant. The translated English transcripts were double checked by the first author (JYL) and any discrepancies were resolved via discussion with another author (SWHL). First, transcripts were coded into an initial set of themes based on keywords or phrases that appeared to convey an opinion or perception regarding telemedicine guided by themes identified from previous literature. ${ }^{11} 1324$ These initial codes were further examined and refined, with codes combined to be main themes if they had similar contexts or split into subthemes via an inductive process. The final codes were summarised, cross-checked and iteratively refined using paired analysis of transcripts by two researchers (JYL and SWHL). In the event that there was a divergent interpretation, the transcripts were reviewed again and discussed until consensus was achieved.

\section{Patient and public involvement}

The current study was designed to understand patients' views, perceptions and experiences in using telemedicine for type 2 diabetes management. Patients were not involved in the initial design of the study, development of interview questions or conduct of the study. All participants in this qualitative study were not provided with the results but were informed that the results will be published in a peer-reviewed journal.

\section{RESULTS}

\section{Demographics of participants}

A total of 48 participants were interviewed in 12 focus groups and two interview sessions. Each focus group had an average of between 3 and 4 participants, as we had difficulty in arranging for larger groups due to different timings of clinic visits. The participants were mostly females $(56.3 \%)$, with a mean age of 51.9 years, and have had diabetes for 5.6 years on average (table 2). Four core themes emerged from the focus groups and the two in-depth interviews: (1) generational difference, (2) independence and convenience, (3) sharing of health data and privacy and (4) concerns and challenges.

\section{Theme 1: generational differences}

Our data suggest that there were generational differences with regard to participants' preference on how to record their blood glucose reading. Generally, older participants in this study (those aged 50 years and above) preferred to record their glucose readings manually, using pen and paper. They felt that the telemonitoring device required technical knowledge and was a challenging and complex process for them. As narrated by the participants:

I (prefer to) manually record (my blood glucose readings). I do not understand (how to operate the mobile) telephone especially operating (the software). To me manual (recording) is easier. (Patient 4, 59/F) 


\begin{tabular}{|c|c|c|}
\hline Patients $(n=48)$ & Men $(n=21)$ & Women $(n=27)$ \\
\hline Age (years) & 54.19 & 50.15 \\
\hline Range & $29-62$ & $31-69$ \\
\hline $\begin{array}{l}\text { Duration of diabetes } \\
\text { (years) }\end{array}$ & 4.06 & 6.88 \\
\hline Range & $0.83-24$ & $0.5-15$ \\
\hline \multicolumn{3}{|c|}{ No of oral hypoglycaemic agents used, n (\%) } \\
\hline $1-2$ & $18(85)$ & $27(100)$ \\
\hline$\geq 3$ & $1(4)$ & $0(0)$ \\
\hline \multicolumn{3}{|c|}{ No of antihypertension drugs used, n (\%) } \\
\hline $1-2$ & $9(42)$ & $11(40)$ \\
\hline$\geq 3$ & $3(14)$ & $7(25)$ \\
\hline \multicolumn{3}{|l|}{ Insulin use, n (\%) } \\
\hline Yes & $4(19)$ & $2(7)$ \\
\hline No & $17(81)$ & $25(93)$ \\
\hline
\end{tabular}

Data are expressed as mean, unless otherwise stated.

But I am an old lady. I like it (blood glucose results) to be written (down). I'm old (and) I need to write (the results) down. Anyway, as long as someone shows me how to do it, I can do it. Of course (using telemedicine) is easier because you bring your hand phone everywhere you go. (Patient 5, 57/F)

These participants felt that the use of telemedicine was more suited for younger individuals with diabetes who were more familiar with technology utilisation. As explained by one participant, 'Maybe for the young ones. Technology (is) for youngsters (and) is more suitable since they like to sit at home and like the thing called Internet'. (Patient 41, 67/M)

Some participants also expressed their preference to meet their healthcare providers in person and reported that travelling to the clinic was preferred.

I don't mind coming to the clinic and have a chat with the doctor. We (can) discuss (my medical condition) and sometimes we can ask questions and doctors can show (my problem to me) physically. For me to use the Internet is difficult to learn (as) my children are not here. (Patient 6, 60/M)

Conversely, younger participants were more inclined to learn and use new technologies if sufficient training and guidance were given. Additionally, they were optimistic about using telemedicine to manage their conditions, as they knew the various benefits that technology would provide, including the data analytics ability and convenience.

\section{Theme 2: independence and convenience}

Participants expressed that the use of a mobile phone connected to a glucometer was a convenient method that contributed to the improved management of their diabetes. Participants considered this to be a convenience when time constraints or logistical issues prevented them from attending the clinic for their appointment. Participants also mentioned that having a telemonitoring device encouraged them to monitor their glucose levels regularly compared with the quarterly check-ups at the clinic. As expressed by participants:

It's good to use especially over the Internet. It's so much easier we do not have to come to the clinic and can stay at home. (Patient 12, 60/M)

Digitals way (is the preferred choice). Everyday you can see (your blood glucose results) in the software so (there is) no need to record like manual. Sometimes even you record manual the paper will go wherever (or) missing. (Using) digital you have a backup. (Patient 8, 35/F)

I like this because you can transfer (your blood glucose results) directly to your phone. It's useful to me as an indication. I prefer (using technology so) that I can use it to check how my medicine affects my glucose. I think that this is the best tools because you can monitor by the Internet everywhere you go. (Patient 9, 44/M)

It's even more convenient since we do not have the time to come (to the clinic) and sometimes some people do not have transport. (Patient 10, 44/F)

Most participants were generally enthusiastic as they saw the potential benefits of telemedicine. Participants described how the use of a web-based glucometer was useful as it could provide them with reminders and alerts as well as the ability to connect with their healthcare providers without going to the clinic. Nevertheless, they expressed the desire and need to have more training and assistance, especially when they had not used the device for some time, since they would have forgotten the functions on the device.

For me it's not difficult ... just need to teach (me) that's all. See the learning condition first (and perhaps I need more training), maybe need two or three times. I'm over fifty (years old) so (the) first time will be a problem .......need to teach a few times before I understand. First time might be difficult to understand. (Patient 7, 56/M)

\section{Theme 3: sharing health data and privacy}

Our analysis showed that there were conflicting perspectives with data sharing. Several participants expressed that they were willing to share their monitoring results with family members, as they felt accountable for their diabetes management, which was a significant motivation for them to meet their daily goals.

The use of this what you call it (web-telemonitor) ......... (I feel) my health is better since my family 
members (also) monitor (my) sugar levels as well. (Patient 11, 54/F)

The good part (of telemedicine is) you can do it at home. If (you check your blood glucose levels) at the clinic you get pricked (but) nobody knows (the results) ... but when you do it at home you children will be informed as well. (Patient 5, 57/F)

Conversely, some participants expressed concern with the sharing of medical information with their family members, whereby they felt that their privacy was infringed and could potentially lead to conflicts between family members.

That thing (Telemonitoring device) is okay (to use) but what I am afraid of is (that the use of telemonitoring may) sometimes (cause) conflicts because it feels like you are being monitored by others. But it is beneficial, at least there is somebody remind you. (Patient 1, 45/M)

Participants also expressed concern about the presence of malware in telemonitoring devices that could compromise their personal information.

(What happens when we have a) problem with the Internet (connection). Occasionally we (may) receive the reading, but occasionally (we will be) unable to (do so). (This can be) very inconvenient. (Patient 11, $54 / \mathrm{F})$

Some participants felt that meeting or contacting their healthcare providers was a simpler and faster way to solve their queries as the healthcare practitioner would be able to explain their clinical measures in a more concise manner. As explained by one participant 'Face-to face (consultation) is more important (as) its better. (With) face-to face (consultation), information is clearer and more satisfying to me'. (Patient 2, 67F)

\section{Theme 4: concerns and challenges}

Participants in this study emphasised the importance of having a user-friendly technology. Most participants in this study reported minor technical difficulties especially with internet connectivity and availability in rural areas, which limited their ability to utilise the telemonitoring device effectively. Participants also expressed concerns regarding the stability of internet connection at their homes.

I think depending on the situation... whether you live in a village where (I feel) it will be very difficult... because (in) certain villages you don't have (Internet) line so you (will) still need have (to record the blood glucose results) manually. (Patient $8,35 / \mathrm{F}$ )

Participants suggested the need to have a robust system which is user-friendly, with good technical support which they felt were essential to ensure the successful implementation of telemedicine.
Okay but what happens when there is a virus? It is a problem for oneweek my handphone "hang". After one week it hangs (again)....that is a problem. (Patient 13, 54/F)

Another aspect which participants were worried of was the costs involved compared with conventional care and that telemonitoring would only be suitable for affluent patients.

All this (telemedicine) is for people who are wealthy. Of course this is a good system. (Patient 14, 63/M)

\section{DISCUSSION}

In our study, we present the conditions in the success of a telemonitoring health device for managing type 2 diabetes from the perspectives of patients. We identified a set of distinctive but interrelated conditions which were and would be central to the success of such programmes in the context of a developing country. First, participants' age were crucial in ensuring the success of telemedicine. We noted that younger participants between the ages of 29 and 50 years old preferred to use a web-based glucometer compared with older participants (aged 50 and above) who preferred to record their results manually. This outcome is not surprising, as younger individuals are more familiar with technology and its use. This was noted in the IDEAS study where the average SMBG uploads were only one reading per week instead of the recommended six readings a week. ${ }^{31}$ Participants cited various reasons for being not being able to use a web-based glucometer. These included the lack of internet connectivity especially in the rural areas. Concerns about the stability of internet connections were the main barrier in using telemedicine in the management of diabetes in this study. The need for a simple, user-friendly technology was also consistently highlighted in order to encourage acceptance and adoption among participants on the use of a remote telemonitoring system.

Participants expressed enthusiasm with the potential added value of telemedicine with to their medical conditions. The perceived benefits of telemedicine included the ability to receive alerts, access to SMBG readings with advice from a healthcare professional; as well as reduced cost ion their treatments. Most participants were willing to incorporate technology as part of their diabetes management, but only if they were confident in using the device or if they could seek help and support easily. Our results support previous research which indicated that personal interaction with healthcare practitioners was important to support people with type 2 diabetes, especially when it involved SMBG. ${ }^{153}$ These views were also expressed by most participants in the present study, and the lack of clinical support especially when communicating through remote telemonitoring led to participants dropping out of the study. 
Living with diabetes can be difficult, especially with the need to comply with a demanding and often confusing set of self-care directives. Many individuals encounter diabetes-related conflicts with family members, which results in strained relationships. As noted in this study, some participants expressed reservation on the feedback and monitoring features of the telemonitoring device, as they felt that the device intruded into their privacy, as well as gave them the feeling of being 'watched'. Consequently, some patients opted not to participate in the IDEAS $^{30}$ randomised controlled trial. The findings of this study identified several salient issues which are reflected in the literature. For example, many others noted that patients accepted telemedicine due to its ability to reduce travel time,$^{34-36}$ increase self-awareness ${ }^{37} 38$ and increase access to care. ${ }^{39} 40$

The present study also found several additional barriers that have not been reported in previous literature, such as conflict between operational practicality versus patient's privacy and healthcare data security. As privacy and security risk may undermine the potential of telemedicine, it is important that software developers must consider this aspect as information security is crucial to support a trusting relationship between patients and providers. Therefore, collaboration with researchers in the field of cybersecurity, especially those specialising in network security and cryptography is necessary.

Our study offers several strengths. By exploring the insights of patients, we illustrate how family values and technology literacy could influence participants' opinions on the use of telemonitoring in their medical condition. This method also allowed us to gain an in-depth understanding and broader views of participants' behaviour when managing diabetes. Our study was also culturally specific in an Asian context.

There were some limitations to our study. First, although we included a diverse sample of participants and reached thematic saturation in our focus groups and in-depth interviews, these participants were only limited to one geographical location in Malaysia. Participants in this current study were recruited from suburban districts in Selangor where connectivity and technology literacy were moderate. Future studies should include patients from both urban and rural locations and also include participants with high technology literacy, as this could potentially influence the uptake and acceptability of telemedicine. While published literature suggests that these trends are likely to be transferable to other regions in Malaysia, the context may differ in other countries and settings. The views expressed here are solely from the patients' and do not represent the views of healthcare providers or policy-makers. Finally, our results cannot draw a definitive conclusion regarding differences in provider practices, patient knowledge as well as attitudes from different clinic sites other than those examined in this study.

\section{Implications for practice}

The continued development and improvement in healthcare technology will hopefully facilitate the use of telemedicine in the future. The largest potential use of telemedicine will be its ability to reduce logistical barriers and hence save time. However, before any telemedicine programmes are introduced, there is a need to consider the economic standing of patients and their access to technology. Those living in rural areas may have limited internet connectivity and hence efforts must be made to ensure that the expectations of patients are met. Also, there is a need to invest in capacity building, especially in human resource. Specifically, the creation of training programmes on the use of telemedicine for both the general public and healthcare workers, are particularly important, especially among the non-information technology competent groups of users.

\section{CONCLUSION}

Results from this study indicate that the implementation of telemonitoring in diabetes management requires strategic planning with input from various stakeholders including, educators, healthcare providers, telecom service providers and patients. The patient's personal and contextual factors, which could have a positive or negative effect on the uptake of the service should be taken into consideration. As such, healthcare providers need to discuss and consider the perspective of their patients to ensure the optimal use of telemedicine to improve patients' clinical parameters and quality of life.

Acknowledgements The authors would like to thank all participants and healthcare providers who contributed their time to share their perspectives with us. We also wish to thank Drs Chirk Jenn $\mathrm{Ng}$ and Chee Piau Wong for helping to edit the article, Chan Yan Yew from the USA and Dr Alan Worsley from Hong Kong University for helping with the English edits of this manuscript.

Contributors All authors named contributed substantially to the document. JYL conducted and transcribed the interview, interpreted the results and wrote the draft manuscript. SWHL obtained the funding, designed the study and provided support in editing the manuscript. CKYC, SSC, TP, KK-CL, NN and CSST contributed to the study design and critical review of the manuscript. All authors approved the final version.

Funding This study was supported by the e-Science fund from the Ministry of Science, Technology and Innovation, Malaysia (03-02-10-SF0238 (MOSTI)), Telemedicine Cluster, Tropical Medicine and Biology platform, Monash University Malaysia (52140757-314-00) and SEGi University Research Fund (SEGi/2013/ SKK/04/1).

Competing interests None declared.

Patient consent for publication All participants provided written informed consent for participation in the research.

Ethics approval The study was approved by the Monash University Research Ethics Committee (CF14/1977-2014001016 and CF15/1073-2015000502) and the National Medical Research Register (NMRR-14-177-19466 and NMRR-14-1368-22943).

Provenance and peer review Not commissioned; externally peer reviewed. Data availability statement Data are available upon reasonable request.

Open access This is an open access article distributed in accordance with the Creative Commons Attribution Non Commercial (CC BY-NC 4.0) license, which permits others to distribute, remix, adapt, build upon this work non-commercially, and license their derivative works on different terms, provided the original work is 
properly cited, appropriate credit is given, any changes made indicated, and the use is non-commercial. See: http://creativecommons.org/licenses/by-nc/4.0/.

ORCID iD

Shaun Wen Huey Lee http://orcid.org/0000-0001-7361-6576

\section{REFERENCES}

1 Ogurtsova K, da Rocha Fernandes JD, Huang Y, et al. IDF diabetes atlas: global estimates for the prevalence of diabetes for 2015 and 2040. Diabetes Res Clin Pract 2017;128:40-50.10.1016/j. diabres.2017.03.024

2 International Diabetes Federation. IDF diabetes atlas. 8th edn. Brussels, Belgium: International Diabetes Federation, 2017.

3 American Diabetes Association. 5. glycemic targets. Diabetes Care 2016;39 Suppl 1:S39-46.

4 Farmer AJ, Perera R, Ward A, et al. Meta-Analysis of individual patient data in randomised trials of self monitoring of blood glucose in people with non-insulin treated type 2 diabetes. BMJ 2012;344.

5 Malanda UL, Welschen LMC, Riphagen II, et al. Self-Monitoring of blood glucose in patients with type 2 diabetes mellitus who are not using insulin. Cochrane Database of Systematic Reviews 2012;13.

6 Schnell O, Barnard K, Bergenstal R, et al. Clinical utility of SMBG: recommendations on the use and reporting of SMBG in clinical research. Diabetes Care 2015;38:1627-33.

7 Machry RV, Rados DV, Gregório GRde, et al. Self-Monitoring blood glucose improves glycemic control in type 2 diabetes without intensive treatment: a systematic review and meta-analysis. Diabetes Res Clin Pract 2018;142:173-87.

8 Chakranon P, Yin Key L, Yee Woon T, et al. Distal technology interventions in people with diabetes: an umbrella review of multiple health outcome. Diabet Med 2019.

9 Zhu H, Zhu Y, Leung S-wai. Is self-monitoring of blood glucose effective in improving glycaemic control in type 2 diabetes without insulin treatment: a meta-analysis of randomised controlled trials. BMJ Open 2016;6:e010524.

10 Cox DJ, Gonder-Frederick L, Ritterband L, et al. Prediction of severe hypoglycemia. Diabetes Care 2007;30:1370-3.

11 Malanda UL, Bot SD, Nijpels G. Self-Monitoring of blood glucose in noninsulin-using type 2 diabetic patients: it is time to face the evidence. Diabetes Care 2013;36:176-8.

12 Lee SWH, Ooi L, Lai YK. Telemedicine for the management of glycemic control and clinical outcomes of type 1 diabetes mellitus: a systematic review and meta-analysis of randomized controlled studies. Front Pharmacol 2017;8:330.

13 Polonsky WH, Fisher L, Schikman $\mathrm{CH}$, et al. Structured selfmonitoring of blood glucose significantly reduces $A 1 c$ levels in poorly controlled, noninsulin-treated type 2 diabetes: results from the structured testing program study. Diabetes Care 2011;34:262-7.

14 Fisher L, Polonsky WH, Parkin CG, et al. The impact of structured blood glucose testing on attitudes toward self-management among poorly controlled, insulin-naïve patients with type 2 diabetes. Diabetes Res Clin Pract 2012;96:149-55.

15 Lee JY, Lee SWH, Nasir NH, et al. Diabetes telemonitoring reduces the risk of hypoglycaemia during Ramadan: a pilot randomized controlled study. Diabetic Medicine 2015;32:1658-61.

16 Lee JY, Wong CP, Tan CSS, et al. Telemonitoring in fasting individuals with type 2 diabetes mellitus during Ramadan: a prospective, randomised controlled study. Sci Rep 2017;7:10119.

17 SWH L, Lee JY, San San Tan C, et al. Strategies to make Ramadan fasting safer in type 2 diabetics: a systematic review and network meta-analysis of randomized controlled trials and observational studies. Medicine 2016;95.

18 Paré G, Jaana M, Sicotte C. Systematic review of home Telemonitoring for chronic diseases: the evidence base. Journal of the American Medical Informatics Association 2007;14:269-77.

19 Paré G, Moqadem K, Pineau G, et al. Clinical effects of home telemonitoring in the context of diabetes, asthma, heart failure and hypertension: a systematic review. J Med Internet Res 2010;12:e21.
20 Hernandez C, Casas A, Escarrabill J, et al. Home hospitalisation of exacerbated chronic obstructive pulmonary disease patients. European Respiratory Journal 2003;21:58-67.

21 Lee SWH, Chan CKY, Chua SS, et al. Comparative effectiveness of telemedicine strategies on type 2 diabetes management: a systematic review and network meta-analysis. Sci Rep 2017;7:12680.

22 Jaana M, Paré G. Home telemonitoring of patients with diabetes: a systematic assessment of observed effects. J Eval Clin Pract 2007;13:242-53.

23 Heckemann B, Wolf A, Ali L, et al. Discovering untapped relationship potential with patients in telehealth: a qualitative interview study. BMJ Open 2016;6:e009750.

24 Scott Kruse C, Karem P, Shifflett K, et al. Evaluating barriers to adopting telemedicine worldwide: a systematic review. J Telemed Telecare 2018;24:4-12.

25 Kruse CS, Krowski N, Rodriguez B, et al. Telehealth and patient satisfaction: a systematic review and narrative analysis. BMJ Open 2017;7:e016242-e42.

26 de Souza CHA, Morbeck RA, Steinman M, et al. Barriers and benefits in telemedicine arising between a high-technology hospital service provider and remote public healthcare units: a qualitative study in Brazil. Telemedicine and e-Health 2017;23:527-32.

27 Koivunen M, Saranto K. Nursing professionals' experiences of the facilitators and barriers to the use of telehealth applications: a systematic review of qualitative studies. Scand J Caring Sci 2018;32:24-44.

28 Mair F, Whitten P. Systematic review of studies of patient satisfaction with telemedicine. BMJ 2000;320:1517-20.

29 Chan CKY, Oldenburg B, Viswanath K. Advancing the science of dissemination and implementation in behavioral medicine: evidence and progress. Int J Behav Med 2015;22:277-82.

30 Lee JY, Chan CKY, Chua SS, et al. Intervention for diabetes with education, advancement and support (ideas) study: protocol for a cluster randomised controlled trial. BMC Health Serv Res 2016;16:524.

31 Lee JY, Chan CKY, Chua SS, et al. Telemonitoring and team-based management of glycemic control on people with type 2 diabetes: a cluster-randomized controlled trial. J Gen Intern Med 2019;94.

32 Nowell LS, Norris JM, White DE, et al. Thematic Analysis:Striving to Meet the Trustworthiness Criteria. International Journal of Qualitative Methods 2017;16.

33 Heisler M, Bouknight RR, Hayward RA, et al. The relative importance of physician communication, participatory decision making, and patient understanding in diabetes self-management. J Gen Intern Med 2002;17:243-52.

34 Müller KI, Alstadhaug KB, Bekkelund SI. Acceptability, feasibility, and cost of telemedicine for nonacute headaches: a randomized study comparing video and traditional consultations. J Med Internet Res 2016;18:e140-e40.

35 Primholdt Christensen N, Danbjørg DB. Use of video consultations for patients with hematological diseases from a patient perspective: qualitative study. J Particip Med 2018;10:e11089.

36 Lee JY, Lee SWH. Telemedicine cost-effectiveness for diabetes management: a systematic review. Diabetes Technol Ther 2018;20:492-500.

37 Piette JD, Marinec N, Gallegos-Cabriales EC, et al. Spanish-speaking patients' engagement in interactive voice response (IVR) support calls for chronic disease self-management: data from three countries. J Telemed Telecare 2013;19:89-94.

38 Whitehead L, Seaton P. The effectiveness of self-management mobile phone and tablet Apps in long-term condition management: a systematic review. J Med Internet Res 2016;18:e97.

39 Polinski JM, Barker T, Gagliano N, et al. Patients' Satisfaction with and Preference for Telehealth Visits. J Gen Intern Med 2016;31:269-75.

40 Anstey Watkins JOT, Goudge J, Gómez-Olivé FX, et al. Mobile phone use among patients and health workers to enhance primary healthcare: a qualitative study in rural South Africa. Soc Sci Med 2018;198:139-47. 